\title{
Optimization of transportation requirements in the deployment of military units
}

\author{
İbrahim Akgün ${ }^{\mathrm{a}, \mathrm{b}}$, Barbaros Ç. Tansel ${ }^{\mathrm{a}, *}$ \\ ${ }^{a}$ Department of Industrial Engineering, Bilkent University, Bilkent 06800, Ankara, Turkey \\ ${ }^{\mathrm{b}}$ Scientific Decision Support Center, Turkish General Staff HQ, Bakanlıklar, Ankara, Turkey
}

Available online 31 August 2005

\begin{abstract}
We study the deployment planning problem (DPP) that may roughly be defined as the problem of the planning of the physical movement of military units, stationed at geographically dispersed locations, from their home bases to their designated destinations while obeying constraints on scheduling and routing issues as well as on the availability and use of various types of transportation assets that operate on a multimodal transportation network. The DPP is a large-scale real-world problem for which analytical models do not exist. We propose a model for solving the problem and develop a solution methodology which involves an effective use of relaxation and restriction that significantly speeds up a CPLEX-based branch-and-bound. The solution times for intermediate-sized problems are around $1 \mathrm{~h}$ at maximum, whereas it takes about a week in the Turkish Armed Forces to produce a suboptimal feasible solution based on trial-and-error methods. The proposed model can be used to evaluate and assess investment decisions in transportation infrastructure and transportation assets as well as to plan and execute cost-effective deployment operations at different levels of planning.
\end{abstract}

(C) 2005 Elsevier Ltd. All rights reserved.

Keywords: Large-scale optimization; Military; Transportation; Mixed integer programming; Deployment; Mobility;

Restriction and relaxation; Branch and bound

\section{Introduction}

In this paper, we study the deployment planning problem (DPP) that may roughly be defined as the problem of the planning of the physical movement of military units, stationed at geographically dispersed

\footnotetext{
* Corresponding author. Tel.: +90312290 1262; fax: +903122664054.

E-mail address: barbaros@ bilkent.edu.tr (B.Ç. Tansel).
} 
locations, from their home bases to their designated destinations while obeying constraints on scheduling and routing issues as well as on the availability and use of various types of transportation assets (TAs) that operate on a multimodal transportation network. Large-scale applications arise in moving military forces at a time of conflict, threat, or crisis. Similar planning needs may also arise for planning the movement of emergency response teams, together with their equipment and supplies, at a time of natural disaster.

The paper describes and discusses various aspects of the DPP and proposes a model for solving it. Solutions are obtained for intermediate-sized problems by an effective use of relaxation and restriction that significantly speed up a CPLEX-based branch-and-bound. Our main contribution is to provide a manageable and solvable model for a large-scale real-world problem for which analytical models are non-existent.

The study is implemented as a part of a capability planning system being developed in the Scientific Decision Support Center of the Turkish General Staff Headquarters. The results are quite encouraging in that the current practice in the planning of a deployment in the Turkish Armed Forces may be reversed from a piece-meal bottom-up approach to an all-encompassing top-down approach.

\section{Problem description}

The DPP involves many military units stationed at various locations, i.e., their home bases. At a time of crisis, a subset of them, which is determined by the nature and extent of the threat under consideration, is required to move to their operation areas (destinations). A call for movement is issued for the active set that specifies among other things the earliest times to depart from home bases, the earliest and latest times to arrive at destinations, and other requirements that must be obeyed during movement.

Deployable items that a unit has are pax (troops) and cargo (weapon systems, equipment, and supplies) which we collectively refer to as items. In the deployment-planning context, a planner deals with welldefined categories of items, e.g., tanks, armored personnel carriers, trucks, and boxes of predefined sizes. In a deployment, a military unit is typically split into three components (advance party, pax party, and cargo party), each of which must be deployed as a whole, e.g., as a convoy. The components may follow different routes. The number and configuration of components of a unit depend on the doctrine, the nature of the threat, available resources, the unit's size, and other relevant factors. There may be certain precedence and/or synchronization requirements between components of a unit. For example, a component may be required to arrive at a location in advance of all other components to "marry-up" with other components at a later time. Similar relations may also be present between different units.

How a unit moves from its home base to its destination depends on the transportation mode selected. Ground transportation, railways, airlift, and shipping lanes are all possibilities. A unit may use one or more of these in succession. If a unit uses a single transportation mode from its origin to its destination, the same set of TAs is used during the entire journey. If a combination of different transportation modes is used, then different sets of TAs are active on that unit at different time intervals. This requires that the unit's items be transferred from one set of TAs to another set at points of connection between different modes. Such points are referred to as transfer points. Main transfer points are harbors, airports, and rail stations. Several zones, e.g., staging and marshalling zones, at transfer points prevent congestion and provide uninterrupted flow of items by providing sufficient space in and adjacent to the terminal area to enable deploying and supporting units to carry out loading/unloading, coordination, control, and preparation operations in harmony. A marshalling area can be regarded as a waiting/parking place and a staging area 
as a service point. In this regard, a capacity may be associated with a transfer point depending on the availability and type of material handling equipment and/or its physical characteristics, e.g., a certain number of docks at a seaport. Similar zones may also be operated at home bases and destinations of units.

A unit may use TAs from three different sources: from its own fleet (organic assets), from military transportation units, and/or from civilian transportation companies, each resulting in a different costing and timing structure. For example, if a TA is outsourced from a commercial company, more time is needed to make it available for its first time usage than an organic asset. Additionally, the leasing cost must be taken into account in outsourcing. Timing issues are key to the scheduling of the movements of units and TAs while costing structure is of prime concern for peacetime decisions. In this regard, the availability of TAs and transportation infrastructure, travel times, loading, unloading, and idle waiting times at home bases, destinations, and transfer points, which all affect the arrival time of a unit at its destination, must be taken into account in the planning.

In determining how many TAs of each type to allocate to a unit, loadability features of TAs are considered. Depending on the types of TAs, the seat, weight, volume, and/or lanemeter capacities of TAs are taken into account. In addition, whether or not the transportation infrastructure supports the movement of both TAs and items with respect to physical characteristics (e.g., width of a tunnel/dock and strength of a bridge), prevailing practices based on current policies, strategies, doctrines, and security concerns must be considered.

In deployment planning, a planner needs to determine the routes to follow, schedule the movements, and allocate the TAs and the transportation infrastructure to the deploying units on a time basis so that all deploying units and their materiel arrive at their destinations at the required times while obeying constraints regarding priorities of the units, availability of resources (TAs, transportation infrastructure, material handling equipment, etc.), capacities, and any other specified issues.

\section{Related literature}

The models that directly address the DPP are grouped under the name of military mobility models. Although there is a concerted effort to develop models in this area for more than 20 years, the literature review shows that the attempts to solve the problem are generally simulation based and that the existing simulation and optimization-based studies address only certain parts of the problem. In this paper, we break away from the existing literature and give an all-encompassing optimization model that deals with all aspects of the DPP simultaneously.

Schank et al. [1] and McKinzie and Barnes [2] review a number of military mobility models. They both state that the major aspect lacking in the models is the use of advanced optimization techniques for estimating force closure and that cumbersome ineffective classical optimization algorithms or simplistic and ineffective greedy approaches are used to find solutions.

Of the military mobility models, the closest one to ours is the study of Rosenthal et al. [3,4] for which the predecessors are Baker [5], Baker and Rosenthal [6], Fuller [7], Goggins [8], Killingsworth et al. [9], Weng [10], Morton et al. [11], Toy [12], Turker [13], Wing et al. [14], and Yost [15].

Rosenthal et al. [3,4] consider only the airlift portion of a deployment operation. They propose a large-scale linear programming model, NRMO (NPS/RAND Mobility Optimizer), to route cargo and troops through a specified network with a given fleet of aircraft subject to many physical and political constraints. The model captures various aspects of an airlift system in a deployment, including aerial 
refueling, tactical aircraft shuttles, and constraints based on crew availability. The authors state that the model is designed to provide insight into issues associated with designing and operating an airlift system but not to provide operational flight schedule recommendations. Some example usages of the model that the authors list are allocating resources that govern the processing capacity of airfields, assessing the relative performance of different mixes of aircraft types, evaluating investment (or divestment) decisions in airfields, and studying roles for aerial refueling aircraft.

NRMO and our model are different from each other with respect to several aspects. The underlying network in our model is a physical transportation network consisting of highways, railways, flight routes, and shipping lanes while in NRMO the network of interest is a simpler one defined by airports and arcs corresponding to direct flights between them. One major difference between our model and NRMO is that moving a deployable unit from its home base to its destination requires determining a route of movement on the physical transportation network in our problem whereas routing decisions are absent in NRMO since direct flights between on- and off-load bases (airports) (with a stopover for refueling as necessary) predetermines the routing structure. Another major difference is the requirement of a convoy formation for movement in our model while no convoy formation is required in NRMO (each flight is a convoy by itself). The scheduling issues encountered in our model is substantially more complicated than in NRMO due to carrier changes at transfer points as well as the presence of possible synchronization and precedence requirements that must be obeyed during movement. Finally, the fact that the underlying network in our model is multimodal causes additional complications in resource allocation at transfer points that arise from the need to handle transfer of items between different TAs of different modes.

We are not aware of any literature that deals with all aspects of the DPP. Despite the fact that there is a rich body of literature on transportation planning (e.g., [16,17] for freight transportation; [18-20] for vehicle routing and scheduling; [21-23] for rail transportation; [24] for ocean transportation; [25] for lessthan-truckload trucking; and [26] for dynamic transportation), the models address only certain parts of the DPP. Just to mention a few important discrepancies from a modeling point of view, consider, for example, the vehicle routing/scheduling and fleet management problems. In these problem classes, a vehicle (truck, jet, container) is assigned to move a load/package from one location to another. From the carrier's point of view, the content of the load is not important. The carrier views the load as a truckload/container or a capacity requirement on a vehicle. In this regard, there is no need to model the movement of the load explicitly; it is sufficient to model only the movement of the vehicle. The DPP, however, requires that the movements of both the load and the vehicle be handled explicitly making sure that they are engaged during movement and disengaged after termination. In these problem classes, a load is not reusable as it disappears from the system after a vehicle is assigned to it. This concept of load makes it unnecessary to assign other vehicles to the same load. In the DPP, on the other hand, a load is reusable and may have to be carried on different types of vehicles at different time intervals. Similarly, assets to deploy in the DPP are reusable as they can be allocated to different loads at different time intervals.

As to the railcar distribution problem, the movement of empty and/or loaded cars on the trains resembles the movement of items on the TAs. However, the existing models for this problem have the following features that make them insufficient to solve the DPP: (1) In most models, the routing of freight cars is not considered explicitly; the flow requirements are generally defined from one terminal to the other. (2) Although train routing, train makeup, and car distribution should be integrated, either the train routing or car flows are accepted as given and one decision is based on the other given one. (3) Timetable for trains, e.g., arrival/departure times of trains and available train capacities, are generally accepted as given. 
Because the loading, unloading, and other operations at yards are included in the timetable, such issues are disregarded in the modeling.

Likewise, the rich body of literature in supply chain management (SCM) does not have any model that address the routing, scheduling, and vehicle assignment aspects of the DPP collectively. For example, in SCM deployment what is modeled is inventory deployment with a focus on the selection of sites at which to store commodities and allocation of customers to these sites. Other location/distribution models are of a similar nature where site selection and demand allocation among server nodes is predominant. Flow problems, including multicommodity flow problems, do not address in any way how to allocate transportation assets among items to be moved between source-destination pairs. In all respects, the DPP appears to stand out as a unique and multifaceted problem for which existing models in the literature fall short of.

\section{Modeling artifacts}

We may view the DPP as posed on a network $\tilde{G}=(\tilde{N}, \tilde{A})$ defined by the union of five subnetworks $\tilde{G}_{i}=$ $\left(\tilde{N}_{i}, \tilde{A}_{i}\right), i=1, \ldots, 5$, corresponding, respectively, to ground, rail, air, sea, and inland-water transportation. We assume each $\tilde{G}_{i}=\left(\tilde{N}_{i}, \tilde{A}_{i}\right)$ is connected and directed. The node and arc sets are defined by $\tilde{N}=\bigcup_{i=1}^{5} \tilde{N}_{i}$ and $\tilde{A}=\bigcup_{i=1}^{5} \tilde{A}_{i}$, where $\tilde{A}_{1}, \ldots, \tilde{A}_{5}$ are assumed to be disjoint. Multiple arcs belonging to the same or different transportation modes between nodes $i$ and $j$ are allowed and differentiated by assigning a different arc number, say $l$, to each arc in the network. Nodes that are common to at least two of the node sets $\tilde{N}_{i}$, are transfer nodes, where a switch occurs in movement from one transportation mode to another.

Let $U=\{1, \ldots, \bar{u}\}$ be the active set of military units that need to be deployed. For each $u \in U$, a source-destination pair $\left(s_{u}, t_{u}\right)$ is specified with $s_{u} \in \tilde{N}$ denoting the home base and $t_{u} \in \tilde{N}$ denoting the designated destination. For convenience, let $N_{\mathrm{S}}=\left\{i \in \tilde{N}\right.$ : node $i$ is an $s_{u}$ for some $\left.u \in U\right\}$ and $N_{\mathrm{D}}=\left\{i \in \tilde{N}_{i}\right.$ : node $i$ is a $t_{u}$ for some $\left.u \in U\right\}$. Some nodes may be both in $N_{\mathrm{S}}$ and in $N_{\mathrm{D}}$. Let $N_{\mathrm{TR}}$ be the set of nodes that are transfer points (harbors, airports, rail stations). We refer to all remaining nodes as transshipment nodes, i.e., $N_{\mathrm{T}}=\tilde{N}-N_{\mathrm{S}} \cup N_{\mathrm{D}} \cup N_{\mathrm{TR}}$, generally used as control points to check the movement of a unit. Define also $A F_{i}$ and $A B_{i}$ to be the forward and backward stars of node $i$, respectively, where $A F_{i}\left(A B_{i}\right)$ consists of arcs whose tails (heads) are at node $i$.

An item list $I_{u}$ that specifies the set of items (personnel, equipment, and supplies) to be moved for unit $u$ is given for each unit $u \in U$. We assume that $I_{u}$ is partitioned into $q(u)$ subsets $I_{u}^{1}, \ldots, I_{u}^{q(u)}$, where each subset defines a deployment component that must be moved as a whole. We remark that an item of a unit may be in different components with different quantities. For each unit $u \in U$, three parameters $e_{u}, a_{u}$, and $b_{u}$ are given specifying, respectively, the earliest time to depart from $s_{u}$ and the earliest and latest times to arrive at $t_{u}$.

For modeling purposes, we assume that all data regarding the to-be-deployed items of all units are arranged in a list as exemplified in Table 2. Each line on the list refers to a particular item that belongs to a particular deployment component of a particular unit. A line specifies the associated item's quantity (in number of units) (column 5), its earliest time to depart from its origin (column 6), its earliest and latest times to arrive at its destination (columns 7 and 8), its dimensions for one unit of it, TAs and parts of the transportation network it can use, and any other related data. As an indexing convention, each deployment component of each military unit is assigned a distinct index $g \in\left\{1,2, \ldots, q^{*}\right\}$, where $q^{*}$ is the total 
Table 1

A portion of an item list $(\tau+\mathrm{h}$ means that item is ready for movement or to be at its destination $\mathrm{h}$ time periods after the day the movement is announced to start)

\begin{tabular}{|c|c|c|c|c|c|c|c|c|}
\hline $\begin{array}{l}\text { Line } \\
\text { index }(c)\end{array}$ & Unit & $\begin{array}{l}\text { Deployment } \\
\text { component }\end{array}$ & Item & Qty. & $\begin{array}{l}e_{c} \\
\text { preptime }_{c}\end{array}$ & $\begin{array}{l}a_{c} \\
\text { mintime }_{c}\end{array}$ & $\begin{array}{l}b_{c} \\
\text { reqtime }_{c}\end{array}$ & $\begin{array}{l}\text { Component } \\
\text { index }(g)\end{array}$ \\
\hline 1 & $\mathrm{~A}$ & Pax & Troop & 200 & $\tau+3$ & $\tau+13$ & $\tau+15$ & 1 \\
\hline 2 & $\mathrm{~A}$ & Cargo & Troop & 50 & $\tau+3$ & $\tau+13$ & $\tau+15$ & 2 \\
\hline 3 & A & Cargo & M-60 Tank & 5 & $\tau+3$ & $\tau+13$ & $\tau+15$ & 2 \\
\hline 4 & A & Cargo & Pax Carrier & 14 & $\tau+3$ & $\tau+13$ & $\tau+15$ & 2 \\
\hline 5 & $\mathrm{~A}$ & Cargo & MRC Truck & 5 & $\tau+3$ & $\tau+13$ & $\tau+15$ & 2 \\
\hline 6 & A & Cargo & $2 \mathrm{~m}^{3}$ Box & 5 & $\tau+3$ & $\tau+13$ & $\tau+15$ & 2 \\
\hline 7 & A & Cargo & $1.5 \mathrm{~m}^{3}$ Box & 10 & $\tau+3$ & $\tau+13$ & $\tau+15$ & 2 \\
\hline 8 & $\mathrm{~B}$ & Pax & Troop & 200 & $\tau+4$ & $\tau+10$ & $\tau+14$ & 3 \\
\hline 9 & B & Cargo & Troop & 50 & $\tau+4$ & $\tau+10$ & $\tau+14$ & 4 \\
\hline 10 & B & Cargo & M-60 Tank & 5 & $\tau+4$ & $\tau+10$ & $\tau+14$ & 4 \\
\hline 11 & B & Cargo & Pax Carrier & 14 & $\tau+4$ & $\tau+10$ & $\tau+14$ & 4 \\
\hline 12 & B & Cargo & MRC Truck & 5 & $\tau+4$ & $\tau+10$ & $\tau+14$ & 4 \\
\hline 13 & B & Cargo & $2 \mathrm{~m}^{3}$ Box & 5 & $\tau+4$ & $\tau+10$ & $\tau+14$ & 4 \\
\hline 14 & B & Cargo & $1.5 \mathrm{~m}^{3}$ Box & 10 & $\tau+4$ & $\tau+10$ & $\tau+14$ & 4 \\
\hline
\end{tabular}

number of components (i.e., $q^{*}=\sum_{u \in U} q(u)$ ). Similarly, each line, i.e., a particular item in possession of a particular component $g$, is assigned a distinct line index $c$. Table 1 illustrates both indexing conventions (columns 1 and 9) on a typical item list. Note that components that belong to the same military unit and item that belong to the same component are consecutively numbered. Most data regarding items are obtained from data regarding units, e.g., the parameters $e_{c}, a_{c}$, and $b_{c}$ are derived from $e_{u}, a_{u}$, and $b_{u}$, respectively, where $c \in I_{u} . \tau$ is the reference time at which the whole deployment activity begins.

The first indexed item in each deployment component $I_{u}^{i}$ is designated as the leader item for that component with the understanding that all other items in that component follow the same route and the schedule as does the leader.

Precedence and/or synchronization requirements between two components $g$ and $g^{\prime}$ of $u$ are assumed to be given in any convenient form (e.g., as a list) and incorporated into the model as side constraints.

For modeling purposes, we define the following item sets: $C$ is the set of all item indices; $C_{g}=\{c \in$ $C: c$ belongs to component $g\} ; C_{\text {pax }}=\{c \in C: c$ refers to personnel/troops $\} ; C_{\text {cargo }}=\{c \in C:$ $c$ refers to items other than troops $\} ; C_{\text {lane }}=\{c \in C: c$ refers to items for which lanemeter capacity is to be taken into account $\} ; C S_{i}=\{c \in C: c$ refers to items whose home base is node $i\} ; C D_{i}=\{c \in C: c$ refers to items whose destination is node $i\} ; C T_{i}=\{c \in C: c$ refers to items that can use node $i$ as a transshipment node $\} ; C T R_{i}=\{c \in C: c$ refers to items that can use node $i$ as a transfer node $\}$; $C P_{i c}=\left\{c^{\prime} \in C: c\right.$ has a lower priority in arriving at node $i$ than $\left.c\right\} ; C F I R S T=\{c \in C: c$ refers to leader items in all components\}.

We assume that TAs that can be used in a deployment are also given as a list. As a convention, each line on the list is given a distinct line index $v$ to refer to TAs of a type (truck, cargo plane, ship, etc.), from a source (organic, common use, civilian), and at a location, i.e., the set of TA types is partitioned into subsets based on location and source type. The indexing convention is illustrated on a portion of a list of TAs in Table 2 . Indexing is done in such a way that all TAs from a particular source type and particular location 
Table 2

A portion of a TA list

\begin{tabular}{|c|c|c|c|c|c|c|c|c|c|c|}
\hline $\begin{array}{l}\text { Line } \\
\text { index }(v)\end{array}$ & Source & Location & TA Type & Qty. & $\begin{array}{l}\text { Load } \\
\text { type }\end{array}$ & $\begin{array}{l}\text { Weight } \\
\text { cap. (ton) }\end{array}$ & Fixed cost (\$) & $\begin{array}{l}\text { Load/ unload } \\
\text { time }\end{array}$ & $\begin{array}{l}\text { Travel time } \\
\text { per }(\mathrm{km})\end{array}$ & $\begin{array}{l}\text { TA Group } \\
(w)\end{array}$ \\
\hline 1 & Unit A & $\mathrm{X}$ & Tank Carrier & 10 & Cargo & 75 & 125,000 & $1 \mathrm{~h}$ & $1.2 \mathrm{~min}$ & 1 \\
\hline 2 & Unit A & $X$ & Truck A & 10 & Cargo & 5 & 50,000 & $1 \mathrm{~h}$ & $1.2 \mathrm{~min}$ & 2 \\
\hline 3 & Unit A & $X$ & Truck B & 5 & Cargo & 10 & 75,000 & $1 \mathrm{~h}$ & $1.2 \mathrm{~min}$ & 2 \\
\hline 4 & Unit B & $X$ & Tank Carrier & 10 & Cargo & 75 & 125,000 & $1 \mathrm{~h}$ & $1.2 \mathrm{~min}$ & 1 \\
\hline 5 & Unit B & $X$ & Truck A & 10 & Cargo & 5 & 55,000 & $1 \mathrm{~h}$ & $1.2 \mathrm{~min}$ & 2 \\
\hline 6 & Unit B & $X$ & Truck B & 5 & Cargo & 10 & 80,000 & $1 \mathrm{~h}$ & $1.2 \mathrm{~min}$ & 2 \\
\hline 7 & Firm A & $\mathrm{Y}$ & Truck A & 5 & Cargo & 5 & 150,000 & $1 \mathrm{~h}$ & $1.2 \mathrm{~min}$ & 2 \\
\hline 8 & Firm A & $\mathrm{Y}$ & Truck B & 5 & Cargo & 10 & 65,000 & $1 \mathrm{~h}$ & $1.2 \mathrm{~min}$ & 2 \\
\hline 9 & Firm A & $\mathrm{Y}$ & Truck C & 5 & Cargo & 10 & 90,000 & $1 \mathrm{~h}$ & $1.2 \mathrm{~min}$ & 2 \\
\hline
\end{tabular}

are consecutively numbered to form a block. Additionally, each line $v$ on the list specifies parameters associated with TA $v$, e.g., the quantity (available number), load type, capacity, ready, loading, unloading, and travel times, fixed and variable costs. Additional columns may also be added to the list as necessary to identify other relevant attributes of TAs. For example, the last column in Table 2 differentiates TAs according to their types only regardless of their location or source type. Such groupings are especially useful in defining capacities on the transportation network.

We define $V=\bigcup_{m=1}^{5} V_{m}$ as the set of TA indices, where $V_{m}, m=1, \ldots, 5$ contains the indices of TAs belonging to transportation mode $m$. In the model, we differentiate transportation modes implicitly by allowing TAs of different transportation modes to move on the appropriate arcs.

For modeling purposes, we define the following TA sets: $V$ is the set of all TA indices; $V_{\operatorname{gr} w}=\{v \in V: v$ refers to TAs in a group $w$ of TAs $\} ; V_{\operatorname{pax}}=\{v \in V: v$ refers to TAs that can carry only personnel $\}$; $V_{\text {cargo }}=\{v \in V: v$ refers to TAs that can carry only cargo $\} ; V_{\text {both }}=\{v \in V: v$ refers TAs that can carry cargo and personnel in separate compartments $\} ; V_{\text {mix }}=\{v \in V: v$ refers to TAs that can carry personnel and cargo in a single compartment $\} ; V_{\text {lane }}=\{v \in V: v$ refers to TAs for which lanemeter capacity is to be taken into account\}.

Additionally, we use the following data regarding items and transportation assets: weight , $_{\text {, }}$ vome $_{c}$, length ${ }_{c}$, demand , paxweight, fixcost $_{v}$, trvcost $_{l_{v}}$, trvcoste $l_{v}$, availVeh $_{v}$, WCap $_{v}$, VCap $_{v}$, PCap $_{v}$, PaxVol $_{v}$, $L C a p_{v}, \operatorname{tr}_{l v}^{\text {loaded }}, \operatorname{trv}_{l v}^{\text {empty }}$, and $r e a d y_{v}$, where the chosen terms are essentially self-explanatory (e.g., weight $_{c}$ is the weight of one unit of item $c$, paxweight is the standard weight of a troop, availveh is the number of units of TA $v$ initially available, $\operatorname{trvcost} f_{l v}$ is the travel cost when a unit of TA $v$ moves loaded on arc $l, P C a p_{v}$ is the passenger capacity of a unit of TA $v$, PaxVol$_{v}$ is the volume consumed by a passenger on a unit of TA $v$, and ready $y_{v}$ is the time necessary for TA $v$ to be ready).

We find it appropriate to point out that $t r v_{l v}^{\text {loaded }}$, the travel time of a unit of TA $v$ on arc $l$ when it is loaded, includes $\operatorname{tr} v_{l v}^{\text {empty }}$, the travel time of a unit of TA $v$ on arc $l$ when it is empty, plus loading and/or unloading times of TA $v$ if the tail and/or head node of the arc is a source, destination, or transfer point.

We assume that the transportation network $\tilde{G}=(\tilde{N}, \tilde{A})$ is node-wise capacitated. Node capacities are expressed in terms of the number of TAs that can pass through the node per unit time and are 


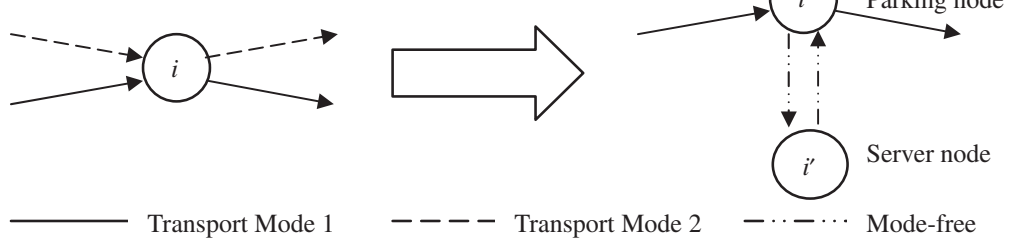

Fig. 1. Second modification of the network.

generally defined by working capacities of the handling and loading/unloading equipment and personnel available at that node. They can be taken as infinity for most nodes, but finite capacities are generally assigned to source, demand, and transfer points as well as to critical nodes such as major intersections and bridges.

The proposed model is arc-wise uncapacitated. Arc capacities can easily be accommodated, if necessary, by introducing artificial dummy nodes on arcs and assigning appropriate node capacities to the artificial nodes.

For modeling purposes, we make two modifications on the network $\tilde{G}=(\tilde{N}, \tilde{A})$. The first modification is to add a single super node $n_{d}$, and a set $A_{\text {dum }}$ of directed $\operatorname{arcs}$ of the form $\left(n_{d}, i\right)$ for each node $i \in \tilde{N}$ that houses at least one TA. The supernode is a dummy node that represents a virtual pool of TAs available anywhere in the network. A given TA with initial location $i$ is drawn from $n_{d}$ for its first time usage through the $\operatorname{arc}\left(n_{d}, i\right)$. A fixed cost fixcost $v_{v}$ and a ready time ready $v \geqslant 0$ (e.g., the contracted time to make a TA available from a civilian company) are associated with TA $v$ drawn from $n_{d}$.

The second modification we make on the network is to add a replica $i^{\prime}$ for nodes $i$ at which loading/unloading operations are expected to take place, i.e., transfer nodes, sources, and destinations (as necessary). Mode-free directed arcs of the form $\left(i, i^{\prime}\right)$ and $\left(i^{\prime}, i\right)$ are also added for each replicated node. The modification is depicted in Fig. 1. It is convenient to view node $i^{\prime}$ as a "server" node, where the actual loading/unloading operations take place, and $i$ as a "parking/waiting lot" for a transportation asset. We define $N_{\mathrm{P}}$ as the set of parking/waiting nodes and refer to server nodes with their original names, i.e., source, demand, and transfer nodes. Define $G=(N, A)$ to be the network obtained from $\tilde{G}=(\tilde{N}, \tilde{A})$ after the dummy nodes and arcs are added to it.

This modification of the network helps to define node capacities and handle timing issues appropriately. If a TA is not to get service at node $i^{\prime}$, it just passes through node $i$ without stopping or spends an idle time, e.g., time to comply with a time-wise constraint, at the node. If a TA is to get service at node $i^{\prime}$, it must move from node $i$ to node $i^{\prime}$ before getting service and from node $i^{\prime}$ to node $i$ after getting service. To incorporate an appropriate service time for a TA at a server node, the associated loading and/or unloading times are taken as travel times of the TA on the $\operatorname{arcs}\left(i, i^{\prime}\right)$ and $\left(i^{\prime}, i\right)$ depending on whether the TA arrives (leaves) at (from) node $i^{\prime}$ empty or loaded. For example, if a TA leaves loaded from node $i^{\prime}$ to node $i$, a non-zero loading time is assigned to the TA, whereas a zero load time is assigned if the TA is empty. If node $i^{\prime}$ is not available for service at any time, the TA spends an idle time at node $i$ until it becomes available. A TA may also spend an idle time at a server node (e.g., waiting for an item to arrive). Such idle times at the nodes are handled by inventory variables appropriately. 
We define the two parameters $\operatorname{Park}_{i w}$ and $\operatorname{Ser}_{i w}$ regarding the transportation network to refer to the parking capacity of node $i$ at a time for TAs in group $w$ and the service capacity of node $i$ at a time for TAs in group $w$, respectively.

From a modeling viewpoint, there are two types of flows on the network: those of items and of TAs. The TAs are the active agents in that the items cannot flow by themselves unless engaged with TAs. In this sense, flows of TAs are coupled for a length of time with flow of items and then disengaged again upon arrival at a destination. We may view everything taking place on a time-expanded network to keep track of scheduling and time-dependent issues associated with movements. We define $T$ to be the set of all time periods and $T D_{c}$ to be the subset of $T$ consisting of time periods at which item type $c$ is allowed to be at a destination. That is, $T D_{c}=\left\{t \in T: a_{c} \leqslant t \leqslant b_{c}\right\}$.

Given the network settings as described above, together with a list of transportation assets and item lists for active units, the DPP involves decisions on (1) the route each deployment component is to follow, (2) the schedule of the movement on this route (departure times from home bases, pass times through transshipment and transfer points, arrival times at destinations, load/unload times at origin, destination, and transfer nodes), (3) the inventory of items at source, demand, and transfer nodes, (4) the TAs and the transportation network each component uses on its route, (5) the load compositions of TAs allocated to each deployment component, (6) the routings of empty TAs subsequent to unloading at destinations, (7) the schedule of the movements of TAs while loaded and empty, (8) the inventory of TAs at origin, transfer, and destination nodes as well as of items of a deployment component at transfer nodes, and (9) a sourcing strategy of TAs for a successful deployment of units.

\section{Model formulation}

We now give a mixed integer programming model for the DPP. Set restrictions that ensure compatibility between TAs and items or TAs and the transportation infrastructure are omitted in the formulation to avoid notational clutter. Such restrictions are assumed present implicitly.

\section{Decision variables}

$T F_{l v t}$ number of units of TA $v$ that start moving loaded on arc $l$ at time $t$,

$T E_{l v t}$ number of units of TA $v$ that start moving empty on $\operatorname{arc} l$ at time $t$,

$C T_{l c v t}$ number of units of item $c$ that start moving on arc $l$ via a unit(s) of TA $v$ at time $t$,

$I V_{i v t}$ the number of units of TA $v$ remaining at node $i$ at time $t$,

$I C_{i c t}$ the number of units of item $c$ remaining at node $i$ at time $t$,

$Y_{l c t} \quad$ zero/one variable which is 1 , if a unit of item $c$ is assigned to start moving on arc $l$ at time $t$ and 0 , otherwise.

\section{Objective function:}

$$
\begin{aligned}
& \underset{T F, T E, C T, I V, I C, Y}{\operatorname{Minimize}} \sum_{l \in A_{\mathrm{dum}}, v, t} \text { fixcost }_{v} \times T E_{l v t}+\sum_{l \in A, v, t} \operatorname{trvcos}_{l} \times T F_{l v t} \\
& +\sum_{l \in A, v, t} \text { trvcoste }_{l v} \times T E_{l v t}
\end{aligned}
$$




\section{Constraints:}

Flow-balance constraints for TAs:

$$
\begin{aligned}
& I V_{i v t}+\sum_{l \in A_{\mathrm{dum}}} T E_{l v t}-I V_{i v, t-1}=0 \quad i=n_{d} v, t \\
& I V_{i v t} \equiv \text { availVeh }_{v} \quad i=n_{d}, v, t=r e a d y_{v} \\
& I V_{i v t}+\sum_{l \in A F_{i}}\left(T F_{l v t}+T E_{l v t}\right)-I V_{i v, t-1}-\sum_{l \in A B_{i}}\left(T F_{l v, t-t r v_{l v}^{\text {loaded }}}+T E_{l v, t-t r v_{l v}^{\text {empty }}}\right)=0 \\
& i \in\left(N-n_{d}\right), v, t
\end{aligned}
$$

Node capacity constraints:

$$
\begin{aligned}
& \sum_{v \in V_{\mathrm{gr} w}} I V_{i v t}+\sum_{l \in A B_{i}, v \in V_{\mathrm{gr} w}}\left(T F_{l v, t-t r v_{l v}^{\text {loaded }}}+T E_{\left.l v, t-t r v_{l v}^{\text {empty }}\right)}\right. \\
& \quad+\sum_{l \in A F_{i}, v \in V_{\mathrm{gr} w}}\left(T F_{l v t}+T E_{l v t}\right) \leqslant \operatorname{Ser} C_{i w} \quad i \in\left(N_{\mathrm{S}} \cup N_{\mathrm{D}} \cup N_{\mathrm{TR}}\right), w, t \\
& \sum_{v \in V_{\mathrm{gr} w}} I V_{i v t} \leqslant \operatorname{Park}_{i w} \quad i \in N_{\mathrm{P}}, w, t
\end{aligned}
$$

Constraints for coupling TAs and items:

$$
\begin{aligned}
& \sum_{c \in C_{\text {cargo }}} \text { weight }_{c} \times C T_{l c v t} \leqslant W C a p_{v} \times T F_{l v t} \quad l \in\left(A-A_{\text {dum }}\right), \quad v \in\left(V_{\text {cargo }} \cup V_{\text {both }}\right), t \\
& \sum_{c \in C_{\text {cargo }}} \text { volume }_{c} \times C T_{l c v t} \leqslant V \operatorname{Cap}_{v} \times T F_{\text {lvt }} \quad l \in\left(A-A_{\text {dum }}\right), \quad v \in\left(V_{\text {cargo }} \cup V_{\text {both }}\right), t \\
& \sum_{c \in C_{\mathrm{pax}}} C T_{l c v t} \leqslant P C a p_{v} \times T F_{l v t} \quad l \in\left(A-A_{\mathrm{dum}}\right), \quad v \in\left(V_{\mathrm{pax}} \cup V_{\mathrm{both}}\right), t \\
& \sum_{c \in C_{\text {lane }}} \text { length }_{c} \times C T_{l c v t} \leqslant L C a p_{v} \times T F_{l v t} \quad l \in\left(A-A_{\text {dum }}\right), \quad v \in V_{\text {lane }}, t \\
& \sum_{c \in C_{\text {cargo }}} \text { weight }_{c} \times C T_{l c v t}+\sum_{c \in C_{\mathrm{pax}}} \text { paxweight } \times C T_{l c v t} \leqslant W C a p_{v} \times T F_{l v t} \\
& l \in\left(A-A_{\text {dum }}\right), \quad v \in V_{\text {mix }}, t \\
& \sum_{c \in C_{\text {cargo }}} \text { volume }_{c} \times C T_{l c v t}+\sum_{c \in C_{\mathrm{pax}}} \text { paxVol }_{v} \times C T_{l c v t} \leqslant V C a p_{v} \times T F_{l v t} \\
& l \in\left(A-A_{\text {dum }}\right), \quad v \in V_{\text {mix }}, t
\end{aligned}
$$


Flow-balance constraints for items:

$$
\begin{aligned}
& I C_{i c t}-I C_{i c, t-1}-\sum_{l \in A B_{i}, v} C T_{l c v t-t r v_{l v}^{\text {loaded }}}=0 \quad i \in N_{\mathrm{D}}, \quad c \in C D_{i} \\
& c \in \text { CFIRST, } t \in T D_{c} \\
& I C_{i c t} \equiv \text { demand }_{c} \quad i \in N_{\mathrm{D}}, \quad c \in C D_{i}, \quad c \in \text { CFIRST }, \quad t=b_{c} \\
& I C_{i c t}+\sum_{l \in A F_{i}, v} C T_{l c v t}-I C_{i c, t-1}=0 \quad i \in N_{\mathrm{S}}, \quad c \in C S_{i}, \quad c \in \text { CFIRST, } \quad t \geqslant e_{c} \\
& I C_{i c t} \equiv \text { demand }_{c} \quad i \in N_{\mathrm{S}}, \quad c \in C S_{i}, \quad c \in \text { CFIRST }, \quad t=e_{c} \\
& I C_{i c t}+\sum_{l \in A F_{i} v} C T_{l c v t}-I C_{i c, t-1}-\sum_{l \in A B_{i}, v} C T_{l c v, t-t r v_{l v}^{\text {loaded }}}=0 \\
& i \in N_{\mathrm{TR}}, \quad c \in C_{i} \quad c \in C F I R S T, t \\
& \sum_{l \in A F_{i}} C T_{l c v t}-\sum_{l \in A B_{i}} C T_{l c v, t-t r v_{l v}^{\text {loaded }}}=0 \quad i \in N_{\mathrm{T}}, \quad c \in C T_{i}, v, t \\
& I C_{i c t}+\sum_{l \in A F_{i}} C T_{l c v t}-I C_{i c, t-1}-\sum_{l \in A B_{i}} C T_{l c v, t-t r v_{l v}^{\text {loaded }}}=0 \quad i \in\left(N_{\mathrm{P}} \cup N_{\mathrm{S}} \cup N_{\mathrm{D}}\right) \\
& c \in\left(C-C S_{i}-C D_{i}\right), v, t
\end{aligned}
$$

Constraints for component unity:

$$
\begin{aligned}
& \left(\sum_{v} C T_{l c v t} / \text { demand }_{c}\right)=Y_{l c t} \quad i \in\left(N-n_{d}\right), \quad l \in A F_{i}, \quad c \in \text { CFIRST, } t \\
& \left(\sum_{v} C T_{l c^{\prime} v t} / \text { demand }_{c^{\prime}}\right)=Y_{l c t} \begin{array}{l}
i \in\left(N-n_{d}\right), \quad l \in A F_{i}, \quad g \in G, \\
c \in C F I R S T, \quad c \in C_{g}, \quad c^{\prime} \in C_{g}, \quad c^{\prime}>c, t
\end{array}
\end{aligned}
$$

Precedence constraints:

$$
\begin{aligned}
& \left(\sum_{v} C T_{l c^{\prime} v, t-t r v_{l v}^{\text {loaded }}} / \text { demand }_{c^{\prime}}\right) \\
& \leqslant\left(\sum_{l^{\prime} \in A B_{i}, v, e_{c} \leqslant t^{\prime}<t} C T_{l^{\prime} c v, t^{\prime}-t r v_{l^{\prime} v}^{\text {loaded }}} / \text { demand }_{c}\right) \quad \begin{array}{l}
i \in N_{\mathrm{D}}, \quad l \in A B_{i}, \quad c \in C F I R S T, \\
c^{\prime} \in C F I R S T, \quad c^{\prime} \in C P_{i c}, t
\end{array}
\end{aligned}
$$


Non-negativity constraints:

$$
\begin{aligned}
& T F_{l v t} T E_{l v t} \geqslant 0 \quad l, v, t \\
& C T_{l c v t} \geqslant 0 \quad l, c, v, t \\
& I V_{i v t} \geqslant 0 \quad i, v, t \\
& I C_{i c t} \geqslant 0 \quad i, c, t \\
& Y_{l c t} \geqslant 0 \quad \text { binary, } l, c, t
\end{aligned}
$$

\section{Initial conditions:}

$$
I T_{i v t} \equiv 0 \quad i \in N_{\mathrm{T}}, v, t
$$

Objective function (1) minimizes the sum of fixed and variable transportation costs. Constraints (2)-(4) ensure the flow-balance of each TA at the supernode and at the remaining nodes for each time period. As an inventory of TAs is not allowed at transshipment nodes, the initial condition (24) is stated accordingly. Constraints (5) and (6) ensure that node capacities are observed at server and parking nodes at each time period. Constraints (7)-(12) are used to couple TAs and items by taking into account seat, weight, volume, and lanemeter capacities on each arc at each time period. Constraints (13)-(19) ensure the flow-balance of items at each node for each time period. Constraints (20) and (21) require that all items in a deployment component move as a whole. Constraints (22) provide precedence relations between items in arriving at a node (between deployment components). Constraints (13)-(17) and (20)-(22) are expressed only for leader items instead of all items. Constraints (21) establish the dependency between leader and non-leader items in each deployment component. Flow-balance constraints (18) and (19) are needed for all items to ensure that the set of TAs assigned to a deployable unit remains intact during the unit's journey except possibly at transfer points.

The decision variables related to items and TAs are allowed to take on fractional values. Among these variables, flow variables $C T_{l c v t}$ may take on fractional values if item $c$ is assigned to two or more TAs. In such a case, the solution is modified so that item $c$ is allocated to its assigned TAs in integral values. Decision variables related to TAs are rounded up when they are fractional. Rounding up retains feasibility while marginally increasing the cost. This is reasonable in the context of the DPP where being economical is secondary to getting the job done (i.e., delivering the items).

The output of the model can be used to determine deployment plans of units and allocation schedules of TAs and transportation infrastructure as follows. It is sufficient to track only the leader item in each deployment component to obtain the route and movement schedule of a deployment component and, hence, of a unit. The values of the decision variables $C T_{l c v t}$ are the main input for this. The time-based allocation of transportation infrastructure to deployment components is obtained from the variables $C T_{l c v t}$ and $I C_{i c t}$. The variables $C T_{l c v t}$ also give a coupling of an item index with a TA index on an arc at given time. Hence, the TAs that are used to carry an item on an arc at a time epoch can be determined. If only one item index $c$ is coupled with a TA index $v$ on an arc at a time epoch, then the value of the decision variable $T F_{l v t}$ on the arc at time $t$ gives the number of units of TA $v$ assigned to carry item $c$. If more than one item is coupled with TA $v$, then the items can be assigned to TAs by taking the capacities of the TAs into account. The values of the decision variables $T E_{l v t}$ give the numbers, routes and schedules of empty TAs. That the values of TA and item inventory variables $I V_{i v t}$ and $C T_{l c v t}$, respectively, are greater than zero at a node shows that TAs and items are waiting idle at that node (e.g., waiting for another deployment 
component to arrive). Because each TA index refers to TAs of a type from a source type at a location, the values of the decision variables $T E_{l v t}$ corresponding to $l \in A_{\text {dum }}$ give a sourcing strategy for TAs.

\section{Computational results for DPM}

Table 3 summarizes the characteristics of the problems generated to test the performance of the DPM. In the test problems, three networks of different sizes are used. The numbers of nodes and arcs are, respectively, 13, 18, 25 and 48, 77, and 109. Five problems are generated for each network by setting the number of item indices to 4, 8, 16,32, and 64. Four deployment components consisting of equal number of item indices are assumed in all problems. The home bases of the components are different for all problems, i.e., four source nodes. There are three destinations for problems 1-10 and four destinations for the remaining ones. A precedence relationship is established between two components in arriving at the destination for problems 1-10. In all problems, the deployment components can use six different indices of TAs that are located at three different locations. A time window of 20 time periods is allocated to units to arrive at their destinations for the smallest-size network and a time window of 40 time periods for the remaining two networks. The time span of the planning is 100 time periods in all problems.

The computational tests are implemented on a $1.5 \mathrm{GHz}$ PIV PC with $1.5 \mathrm{~GB}$ RAM by using ILOG CPLEX 9.0 and by letting the models run until the desired optimality criterion is attained or for $8 \mathrm{~h}$ $(28,800 \mathrm{CPU}$ s) at maximum. The solution times at which the root node (LP relaxation) is solved and $10 \%, 5 \%$, and $0 \%$ deviation from optimality are achieved are recorded in all computational studies. Table 4 gives the solution times of the DPM for problems 1-5 where constraints (2) and (3) are not used, i.e., an infinite number of TAs of each index is assumed. Table 4 shows that the solution times of DPM based on a direct use of CPLEX 9.0 are not good enough to be used in a real-world application. The optimal solutions of the DPM are obtained only for problems 1 and 2 in around 5000 CPU s. However,

Table 3

Characteristics of the generated test problems

\begin{tabular}{|c|c|c|c|c|c|c|c|c|c|}
\hline Pr. Id. & $|N|$ & $|A|$ & $\left|N_{\mathrm{S}}\right|$ & $\left|N_{\mathrm{D}}\right|$ & $|G|$ & $|C|$ & $|V|$ & $|T D|$ & $|T|$ \\
\hline 1 & 13 & 49 & 4 & 3 & 4 & 4 & 6 & 20 & 100 \\
\hline 2 & 13 & 49 & 4 & 3 & 4 & 8 & 6 & 20 & 100 \\
\hline 3 & 13 & 49 & 4 & 3 & 4 & 16 & 6 & 20 & 100 \\
\hline 4 & 13 & 49 & 4 & 3 & 4 & 32 & 6 & 20 & 100 \\
\hline 5 & 13 & 49 & 4 & 3 & 4 & 64 & 6 & 20 & 100 \\
\hline 6 & 19 & 77 & 4 & 3 & 4 & 4 & 6 & 40 & 100 \\
\hline 7 & 19 & 77 & 4 & 3 & 4 & 8 & 6 & 40 & 100 \\
\hline 8 & 19 & 77 & 4 & 3 & 4 & 16 & 6 & 40 & 100 \\
\hline 9 & 19 & 77 & 4 & 3 & 4 & 32 & 6 & 40 & 100 \\
\hline 10 & 19 & 77 & 4 & 3 & 4 & 64 & 6 & 40 & 100 \\
\hline 11 & 25 & 109 & 4 & 4 & 4 & 4 & 6 & 40 & 100 \\
\hline 12 & 25 & 109 & 4 & 4 & 4 & 8 & 6 & 40 & 100 \\
\hline 13 & 25 & 109 & 4 & 4 & 4 & 16 & 6 & 40 & 100 \\
\hline 14 & 25 & 109 & 4 & 4 & 4 & 32 & 6 & 40 & 100 \\
\hline 15 & 25 & 109 & 4 & 4 & 4 & 64 & 6 & 40 & 100 \\
\hline
\end{tabular}


Table 4

Solution times of DPM (CPU seconds)

\begin{tabular}{|c|c|c|c|c|}
\hline \multirow[t]{2}{*}{ Pr. Id. } & \multicolumn{4}{|l|}{ DPM } \\
\hline & Root & $10 \%$ & $5 \%$ & $0 \%$ \\
\hline 1 & 10.42 & - & - & 48.67 \\
\hline 2 & 267.22 & 3623.99 & - & 4536.65 \\
\hline 3 & 2511.03 & - & - & 5182.31 \\
\hline 4 & $11,980.34$ & No integer solution & & \\
\hline 5 & No root solution & & & \\
\hline
\end{tabular}

A " - " for a corresponding optimality criterion shows that the branch-and-bound jumps to a solution with a lower optimality criterion.

even the root solutions cannot be obtained for problems 4 and 5 in the allocated time. Hence, a solution methodology to improve the solution times of the DPM is needed for real-world applications. In what follows, we give the proposed methodology and the solution times based on it .

\section{Proposed solution methodology}

The proposed solution methodology includes finding a relaxation and restriction of the DPM and then using their solutions in solving the DPM. The procedure works as follows: (1) If the relaxation DPM-REL is solved to optimality, then set the lower-bound $z_{\mathrm{DPM}}$ of DPM to the optimal value $z_{\mathrm{REL}}^{*}$ of the DPMREL. Otherwise, set $z_{\mathrm{DPM}}$ to a lower-bound $z_{\mathrm{REL}}$ of $z_{\mathrm{REL}}^{*}$ (2) Use the solution $x_{\mathrm{RES}}^{*}$ of the DPM-RES as a starting solution of the DPM. The objective function value corresponding to $x_{\mathrm{RES}}^{*}$ is an upper bound on the optimal objective function value of the DPM. The details of the solution methodology are depicted in Fig. 2.

DPM-REL is obtained by restricting some of the constraint defining sets to their subsets, thereby deleting the related constraints. Specifically, we redefine constraints (7)-(12) and (20)-(21) for $l \in$ $\left(A_{\text {out }} \cup A_{\text {in }}\right)$ and constraints (18) and (19) for $c \in C F I R S T$. Here, $A_{\text {out }}=\bigcup_{i \in\left(N_{\mathrm{S}} \cup N_{\mathrm{D}} \cup N_{\mathrm{TR}}\right)} A F_{i}$ and $A_{\text {in }}=\bigcup_{i \in\left(N_{\mathrm{S}} \cup N_{\mathrm{D}} \cup N_{\mathrm{TR}}\right)} A B_{i}$ are the sets of arcs outgoing from source, demand, and transfer nodes and incoming to source, demand, and transfer nodes, respectively.

The DPM-RES is obtained by fixing the time periods at which deployment components arrive at destinations and transfer points. The DPM-RES is formulated by fixing the values of the decision variables $Y_{l c t}$ that correspond to $l \in A_{\text {in }}$ and $I C_{i c t}$ in the DPM. In deciding the values of the decision variables to fix, the solution of the DPM-REL is used. The values of the decision variables $Y_{l c t}, l \in A_{\text {in }}$, and $I C_{i c t}$ in the solution $x_{\text {REL }}$ of DPM-REL are set to the values of the corresponding decision variables in the DPM. The DPM-RES determines the routes and schedules of the deployment components and the allocation of TAs and transportation infrastructure given the arrival times of the deployment components at destinations. Hence, the DPM-RES is solved to optimality in a very short time.

One main question is whether the DPM-RES may be infeasible or not. There is a possibility of infeasibility when the number of TAs is not sufficient to make the deployment components ready at their fixed times. However, this is a strong indication that the DPM itself is infeasible because the availability of TAs is checked in the DPM-REL. 


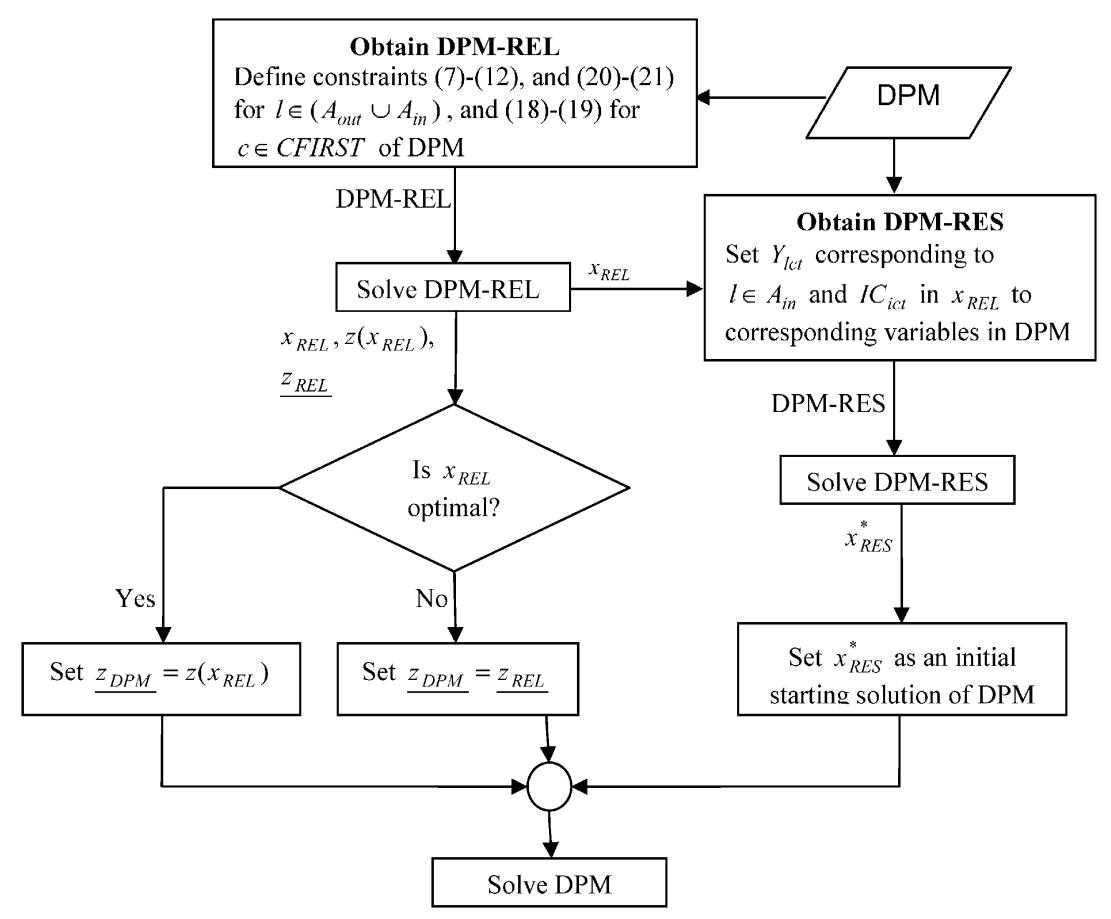

Fig. 2. The depiction of solution methodology.

\section{Computational results for the proposed solution methodology}

We test the performance of the proposed solution methodology using the test problems 1-15 defined in Table 3. The model is tested for both an unlimited and limited supply of TAs. Tables 5 and 6 give the solution times in CPU seconds for unlimited and limited fleet sizes, respectively, for DPM-REL and DPM-RES. The solution times for DPM are not presented because the optimal objective function values of the DPM-RES are either equal to or slightly greater than (the difference is less than $0.01 \%$ ) those of the DPM-REL and the optimal solutions of DPM-RES are feasible for DPM for all problems. That is, the optimal solutions of the DPM- RES are also optimal for the DPM.

The results in Tables 5 and 6 can be considered in two groups, one for the results of problems 1-5 and the other for the results of problems 6-15. The total solution times for the latter group range from 119.51 (Pr. 6) to 1081.17 (Pr. 15) CPU seconds in Table 5 and from 193.17 (Pr. 6) to 4005.64 (Pr. 13) CPU seconds in Table 6. On the average, the solution times of the DPM-REL account for more than $98 \%$ of the total solution times. The times at which the root solution and the optimal solution of the DPM-REL are reached are very close to each other because the optimal solutions are obtained at the root node or after fathoming a small number of nodes in the branch-and-bound algorithm. No solution times of the DPM-REL are presented in the fields corresponding to the optimality criteria of $10 \%$ and $5 \%$ because the branch-and-bound jumps directly to the optimal solution from the first integer solution with a 20-30\% gap between the lower and upper bounds.

The total solution times for problems 1-5 range from 201.81 (Pr. 3) to 800.68 (Pr. 2) CPU seconds in Table 5 and from 503.27 (Pr. 3) to 1410.55 (Pr. 1) CPU seconds in Table 6. As in problems 6-15, the 
Table 5

Solution times of DPM-REL and DPM-RES (CPU seconds)

\begin{tabular}{|c|c|c|c|c|c|c|}
\hline \multirow[t]{2}{*}{ Pr. Id. } & \multicolumn{4}{|c|}{ DPM-REL } & \multirow{2}{*}{$\begin{array}{l}\text { DPM-RES } \\
0 \%\end{array}$} & \multirow[t]{2}{*}{ Total solution time } \\
\hline & Root & $10 \%$ & $5 \%$ & $0 \%$ & & \\
\hline 1 & 10.64 & 348.21 & 373.37 & 405.91 & 7.69 & 413.60 \\
\hline 2 & 67.94 & - & - & 792.12 & 8.56 & 800.68 \\
\hline 3 & 47.25 & - & 54.40 & 197.17 & 4.64 & 201.81 \\
\hline 4 & 92.48 & - & 97.88 & 553.61 & 5.63 & 559.24 \\
\hline 5 & 112.92 & - & 115.437 & 574.10 & 4.28 & 578.38 \\
\hline 6 & 80.67 & - & - & 115.60 & 3.91 & 119.51 \\
\hline 7 & 314.23 & - & - & 362.34 & 5.08 & 367.42 \\
\hline 8 & 456.25 & - & - & 514.73 & 6.97 & 521.70 \\
\hline 9 & 344.76 & - & - & 457.54 & 12.90 & 470.44 \\
\hline 10 & 609.78 & - & - & 612.36 & 23.94 & 636.30 \\
\hline 11 & 176.60 & - & - & 472.06 & 6.68 & 478.74 \\
\hline 12 & 567.06 & - & - & 854.23 & 5.78 & 860.01 \\
\hline 13 & 562.66 & - & - & 565.47 & 12.07 & 577.54 \\
\hline 14 & 554.52 & - & - & 557.93 & 22.35 & 580.28 \\
\hline 15 & 805.44 & - & - & 1035.90 & 45.27 & 1081.17 \\
\hline
\end{tabular}

A “_" for a corresponding optimality criterion shows that the branch-and-bound jumps to a solution with a lower optimality criterion.

Table 6

Solution times of DPM-REL and DPM-RES (CPU seconds)

\begin{tabular}{|c|c|c|c|c|c|c|}
\hline \multirow[t]{2}{*}{ Pr. Id. } & \multicolumn{4}{|c|}{ DPM-REL } & \multirow{2}{*}{$\begin{array}{l}\text { DPM-RES } \\
0 \%\end{array}$} & \multirow[t]{2}{*}{ Total solution time } \\
\hline & Root & $10 \%$ & $5 \%$ & $0 \%$ & & \\
\hline 1 & 12.834 & 961.50 & 1098.66 & 1404.79 & 5.76 & 1410.55 \\
\hline 2 & 96.55 & - & - & 1270.85 & 7.21 & 1278.06 \\
\hline 3 & 109.75 & 115.42 & - & 499.82 & 3.45 & 503.27 \\
\hline 4 & 86.27 & 98.36 & - & 572.47 & 4.43 & 576.90 \\
\hline 5 & 153.95 & 156.39 & - & 965.26 & 6.87 & 972.13 \\
\hline 6 & 130.17 & - & - & 182.10 & 11.07 & 193.17 \\
\hline 7 & 498.36 & - & - & 519.33 & 16.02 & 535.35 \\
\hline 8 & 918.48 & - & - & 935.56 & 28.07 & 963.63 \\
\hline 9 & 836.21 & - & - & 881.91 & 46.44 & 928.35 \\
\hline 10 & 1169.78 & - & - & 1203.05 & 98.48 & 1301.53 \\
\hline 11 & 253.70 & - & - & 2983.39 & 21.99 & 3005.38 \\
\hline 12 & 1597.69 & - & - & 3755.53 & 31.44 & 3786.97 \\
\hline 13 & 2881.34 & - & - & 3959.07 & 46.57 & 4005.64 \\
\hline 14 & 2238.60 & - & - & 2310.08 & 84.79 & 2394.87 \\
\hline 15 & 2276.52 & - & - & 2375.29 & 191.33 & 2566.62 \\
\hline
\end{tabular}

A "_-" for a corresponding optimality criterion shows that the branch-and-bound jumps to a solution with a lower optimality criterion. 
solution times of the DPM-REL account for more than $98 \%$ of the total solution times. However, unlike in problems 6-15, the times at which the root solutions and the optimal solutions of the DPM-REL are reached are not close. The upper bounds, i.e., the first integer solutions, are very close to the optimal solutions and the branch-and-bound algorithm finds a first integer solution in a very short time (in seconds) after the root solution. However, the branch and bound spends around $90 \%$ of the total solution times to increase the lower bounds. This combined with the results for problems 6-15 leads one to think that the optimal solutions are close to the upper bounds.

These results show that the DPM-REL and the DPM-RES obtained from the solution of the DPM-REL provide so good a lower bound and an upper bound, respectively, that an optimal (near-optimal) solution to the DPM can be obtained without having to solve the DPM. Why the DPM-REL provides so good a bound is explained as follows. In the DPM, the movement of a component as whole, the coupled movements of items of a component and TAs, and tracking the movements of all items of a component are required on all arcs from origin to destination of a component. In the DPM-REL, these are required only on the arcs adjacent to origin, destination, and possible transfer points of a component. In this regard, the number of TAs used to deploy a component at the origin and transfer points of a component is essentially the same in both DPM and DPM-REL; however, the routes they follow in DPM and DPM-REL may differ. With respect to cost, this means that fixed costs incurred in both DPM and DPM-REL are essentially the same; however, variable costs may differ. Thus, the objective function values of DPM and DPM-REL are close to each other as their objective functions are the same. What is done in the DPM-RES is actually to correct the relaxed requirements. In this regard, we can mainly focus on improving the solvability of the DPM-REL.

The solution times in Table 6 are worse than those in Table 5. This is expected. The worst case is for problem 13 where the solution time in Table 5 is about one-seventh of its corresponding solution time in Table 6 while the best case is for problem 4 where the solution time in Table 5 is about $97 \%$ of its corresponding solution time in Table 6.

There are no preceding results to compare with ours. However, solution times in Tables 5 and 6 are really encouraging for a real-world application as it is known by experience that it may take a planner about one week to come up with a feasible, not optimal, detailed deployment plan for the size of the problem that we deal with.

The solution times in all tables are obtained under certain parameter settings of the CPLEX. Our experience shows that using the bound strengthening has the effect of worsening the solution times almost 10 times. Using cuts also has an adverse effect on the solution times. The aggressive scaling parameter improves the solution times of the DPM-REL but worsens those of the DPM-RES and the DPM. The algorithm used to solve the LP relaxations at the nodes is primal simplex for the DPMREL and DPM and dual simplex for the DPM-RES. The steepest-edge pricing is good for the DPMREL while the devex pricing is good for the DPM-RES and the DPM. In addition, best-bound node strategy in selecting a node and pseudocosts in selecting branching variable improve the solution times of the models.

\section{Conclusion}

In this paper, we have studied the DPP which is the problem of the planning of the physical movement of military units, stationed at geographically dispersed locations, from their home bases to their designated destinations while obeying constraints on scheduling and routing issues as well as on the availability 
and use of the transportation infrastructure and of various types of TAs that operate on a multimodal transportation network. Large-scale applications of the DPP arise in moving military forces at a time of conflict, threat, or crisis or in moving emergency response teams, together with their equipment and supplies, at a time of natural disaster.

We are not aware of any model in the literature that deals with all aspects of the DPP. In the paper, we propose an all-inclusive model for the DPP and provide a solution methodology that involves an effective use of a relaxation and restriction strategy that significantly speeds up a CPLEX-based branch and bound. The solution times for intermediate-sized problems are around $1 \mathrm{~h}$ at maximum. The model can be used for evaluating and assessing investment decisions in transportation infrastructure and TAs as well as for planning and execution of cost-effective deployment operations at different levels of detail.

\section{References}

[1] Schank J, Mattock M, Sumner G, Greenberg I, Rothenberg J, Stucker JP. A review of strategic mobility models and analysis. Report R-3926-JS, RAND Corporation, Santa Monica, CA; 1991.

[2] McKinzie K, Barnes JW. A review of strategic mobility models supporting the defense transportation system. June 2003. (http://www.me.utexas.edu/ barnes/research/files/update_06_2003/RSMMSDTS.pdf)

[3] Baker SF, Morton DP, Rosenthal RE, Williams LM. Optimizing strategic airlift. Technical Report NPS-OR-99-004, Naval Postgraduate School, Monterey, CA; 1999.

[4] Baker SF, Morton DP, Rosenthal RE, Williams LM. Optimizing military airlift. Operations Research 2002;50(4):582-602.

[5] Baker SF. A cascade approach for staircase linear programs with an application to air force mobility. PhD dissertation, Naval Postgraduate School, Monterey, CA; 1997.

[6] Baker SF, Rosenthal RE. A cascade approach for staircase linear programs. Technical Report NPS-OR-98-004, Naval Postgraduate School, Monterey, CA; 1998.

[7] Fuller D. Reduction of a large-scale global mobility optimization model through aggregation. Master's thesis, Naval Postgraduate School, Monterey, CA; 1994.

[8] Goggins D. Stochastic modeling for airlift mobility. Master's thesis, Naval Postgraduate School, Monterey, CA; 1995.

[9] Killingsworth P, Henry K, Melody L, Stucker J. Tankers: air mobility roles for the 1990s. Report DRR-913-AF, Rand Corporation, Santa Monica, CA; 1994.

[10] Weng LT. Strategic airlift optimization model. Master's thesis, Naval Postgraduate School, Monterey, CA; 1994.

[11] Morton DP, Rosenthal RE, Weng LT. Optimization modeling for airlift mobility. Military Operations Research 1996;1: 49-68.

[12] Toy AO. Route prioritization for an airlift mobility optimization model. Master's thesis, Naval Postgraduate School, Monterey, CA; 1996.

[13] Turker Y. Route and column generation methods for airlift mobility optimization. Master's thesis, Naval Postgraduate School, Monterey, CA; 1995.

[14] Wing VF, Rice RE, Sherwood R, Rosenthal RE. Determining the optimal mobility fix. Joint Staff (J8), Force Design Division, The Pentagon, Washington, DC; 1991.

[15] Yost YA. The THRUPUT strategic airlift flow optimization model. Air Force Studies and Analyses Agency, The Pentagon, Washington, DC; 1994.

[16] Crainic T, Laporte G. Planning models for freight transportation in design and operation of civil and environmental engineering systems. New York: Wiley Interscience; 1997. p. 343-94.

[17] Crainic T. A survey of optimization models for long-haul freight transportation. In: Hall RW, editor. Handbook of transportation science 2nd ed. Dordrecht: Kluwer; 2003. p. 415-516.

[18] Bodin L, Golden B, Assad A, Ball M. Routing and scheduling of vehicles and crews. Computers and Operations Research 1983;10(2):63-211.

[19] Fisher M. Vehicle routing. In: Monma C, Magnanti T, Ball M, editors. Handbook in operations research and management science, volume on networks. Amsterdam: North-Holland; 1995. p. 1-33. 
[20] Desrosiers J, Solomon M, Soumis F. Time constrained routing and scheduling. In: Monma C, Magnanti T, Ball M, editors. Handbook in operations research and management science, volume on networks. Amsterdam: North-Holland; 1995. p. 35-139.

[21] Haghani A. Formulation and solution of a combined train routing and make-up, and empty-car distribution model. Transportation Research 1989;23B(6):433-52.

[22] Glickman T, Sherali H. Large-scale network distribution of pooled empty freight cars over time, with limited substitution and equitable benefits. Transportation Research 1985;19:85-94.

[23] Crainic T, Ferland J, Rousseau JM. A tactical planning model for rail freight transportation. Transportation Science 1984;18(2):165-84.

[24] Brown G, Graves G, Ronen D. Scheduling ocean transportation of crude oil. Management Science 1987;33:335-46.

[25] Crainic T, Roy J. Design of regular intercity driver routes for the LTL motor carrier industry. Transportation Science 1992;26:280-95.

[26] Powell WB. Dynamic models of transportation operations. In: Handbooks in operations research volume on supply chain management; October 2002. 